\title{
Wave propagation in one-dimensional fluid-saturated porous metamaterials
}

\author{
Yan-Feng Wang, ${ }^{1,2}$ Jun-Wei Liang, ${ }^{2}$ A-Li Chen, ${ }^{2}$ Yue-Sheng Wang, ${ }^{1,}{ }^{*}$ and Vincent Laude ${ }^{3, \dagger}$ \\ ${ }^{1}$ School of Mechanical Engineering, Tianjin University, 300350 Tianjin, China \\ ${ }^{2}$ Institute of Engineering Mechanics, Beijing Jiaotong University, 100044 Beijing, China \\ ${ }^{3}$ Institut FEMTO-ST, Université Bourgogne Franche-Comté, CNRS, 25030 Besançon, France
}

\begin{abstract}
Fluid-saturated porous metamaterials described following Biot's theory support two longitudinal elastic waves. The phase velocity and attenuation of these waves depend non linearly on porosity and viscosity of the fluid. Furthermore, when two fluid-saturated porous metamaterials are arranged to form a periodic composite, different bandgaps are opened for the two longitudinal waves and these couple to form anti-crossings in the dispersion relation. The complex band structure of one-dimensional composites is derived and compared with numerical transmission through a finite sample obtained by the finite element method. It is found that the anti-crossings disappear rapidly as viscosity increases, while attenuation bandgaps become dominated by the fastest of the two longitudinal waves. Increasing porosity further leads to wider and lower frequency bandgaps. These results are relevant to practical applications of fluid-saturated porous metamaterials, e.g. to engineered soils.
\end{abstract}

\section{INTRODUCTION}

Wave propagation in geological materials or ground ${ }^{1}$ has received considerable attention because of its practical importance in various fields such as earthquake engineering, soil dynamics, geophysics, hydrology, etc. Most existing studies deal with ground vibrations caused by mechanical operation or seismic waves ${ }^{2}$. A great deal of research has already been conducted on constructing wave barriers operating between the vibration source and the protected structures ${ }^{3}$. More recently, periodic wave barriers, inspired by the concept of phononic crystal (PC), have attracted more and more attention ${ }^{4}$. PCs are spatially periodic composites composed of different materials ${ }^{5}$. They can exhibit frequency bandgaps in their transmission spectrum, where the propagation of acoustic or elastic waves is fully prohibited. The PC concept was proposed in 1993, although there had been many works about wave propagation in periodic media and structures before $^{6}$. In 2000, Liu et al. ${ }^{7}$ further introduced the concept of local resonance inducing complete bandgaps at low frequencies and providing a negative dynamic effective mass density inside resonant bandgaps ${ }^{8}$. Locally-resonant PCs are also termed acoustic/elastic metamaterials ${ }^{9}$, since their band gaps are not significantly dependent on periodicity. PCs and metamaterials provide new ways to manipulate acoustic/elastic waves. Bandgap engineering ${ }^{10}$ ambitions to realize control over wave propagation.

A direct application of bandgap engineering is noise isolation and vibration reduction. Niousha and Motosaka ${ }^{4}$ investigated the effects of periodic wave barriers on the reduction of ground vibration. Jia and Shi ${ }^{11}$ studied the influence of physical and geometrical parameters of periodic foundation on the bandgaps. Malcolm and Nicholls ${ }^{12}$ examined the scattering of periodic multilayered media. Bao et $a l .{ }^{13}$ discussed the dynamic response of a seven-storey frame structure with periodic foundations. Kim and Das ${ }^{14}$ proposed an earthquake-resistant design by constructing a shell-type waveguide composed of many Helmholtz resonators. Shi and Huang ${ }^{15}$ described the feasibility of reducing seismic waves by locally resonant metamaterials. Mitchell et $a l .{ }^{16}$ proposed a metaconcrete composed of designed aggregates to enhance dynamic performance. Colombi et $a l .{ }^{17}$ designed a seismic metawedge to convert destructive Rayleigh seismic waves into harmless bulk shear waves. Colquitt et al. ${ }^{18}$ carefully analyzed the canonical problem of an array of sub-wavelength resonators placed on either a thin elastic plate or an elastic half-space. On the experimental side, a full-scale experiment aiming at researching the interaction of structured soil with seismic waves was realized by Brûlé et al. ${ }^{19}$. Yan et al. ${ }^{20}$ conducted field experiments of a scaled periodic foundation. Colombi et $a l .{ }^{21}$ demonstrated experimentally that a Rayleigh wave experiences strong attenuation when interacting with a forest acting as a locally resonant metamaterial.

Although numerous theoretical studies have explored the properties of PCs and metamaterials, they often consider soil as an elastic constituent, within which waves furthermore often propagate without attenuation. Some studies of PCs or metamaterials composed of fluidsaturated porous (FSP) media have emphasized their specific acoustic properties ${ }^{22-25}$ and have also discussed wave attenuation in FSP media ${ }^{26}$. Actually, wave propagation in FSP media has attracted significant attention for a long time ${ }^{27-30}$. The related theory is of great significance in the fields of geophysical exploration, seismic engineering, or geodynamics. Biot ${ }^{31,32}$ formulated the basic equations for isotropic FSP media. It follows from this theory that two longitudinal and two transverse waves exist. Plona ${ }^{33}$ and Dutta ${ }^{34}$ later proved this fact: the speed of the slow compressional wave is smaller than the speed of sound in the fluid. Wave propagation in FSP media has since then been investigated from different viewpoints and using different methods ${ }^{35-39}$. The effect on the propagation of elastic waves of the material parameters of the fluid, the solid skeleton, and of their combination was discussed. Slowness surfaces ${ }^{38}$ and wave fronts ${ }^{40}$ were calculated by using plane-wave theory or characteristic 
analysis. We note that previous investigations of PCs involving FSP media ${ }^{22-26}$ have not specifically considered the possible interference of the two longitudinal waves.

In this paper, we conduct a detailed analysis of the dynamical behavior of wave propagation in one-dimensional fluid-saturated porous metamaterials (FSPMs). Of particular interest is the mutual interaction of the two longitudinal acoustic waves in FSP media and how they attenuate differently in FSPMs. The basic equations are first summarized in Section II. Harmonic Bloch wave propagation and its finite element implementation are discussed in Section III. These equations are used in Section IV to obtain dispersion relations. To start with, we study wave propagation in homogeneous FSP media. We then consider the effect of viscosity and porosity on the complex band structure and the frequency response function of one-dimensional FSPMs. Only normal incidence of longitudinal waves is considered in this paper. Extensions of our approach to general incidence and more periodicities are suggested in the conclusion.

\section{BASIC EQUATIONS}

Following Biot ${ }^{31,32}$, the constitutive equations for wave propagation in an isotropic FSP media can be written

$$
\begin{array}{r}
\tau_{x x}=\left(2 B_{1}+B_{2}\right) e_{x x}+B_{2} e_{y y}+B_{2} e_{z z}+B_{3} \xi \\
\tau_{y y}=B_{2} e_{x x}+\left(2 B_{1}+B_{2}\right) e_{y y}+B_{2} e_{z z}+B_{3} \xi \\
\tau_{z z}=B_{2} e_{x x}+B_{2} e_{y y}+\left(2 B_{1}+B_{2}\right) e_{z z}+B_{3} \xi \\
\tau_{y z}=2 B_{1} e_{y z}, \tau_{z x}=2 B_{1} e_{z x}, \tau_{x y}=2 B_{1} e_{x y}, \\
p=B_{3} e_{x x}+B_{3} e_{y y}+B_{3} e_{z z}+B_{4} \xi .
\end{array}
$$

In these expressions, $\tau_{i j}$ and $e_{i j}(i, j=x, y, z)$ are the stress and strain tensors of the solid skeleton, $p$ is the pore fluid pressure, and $\xi$ is the increment of the fluid content per unit volume. The displacement components of the skeleton and of the fluid are $u_{i}$ and $U_{i}$. All these quantities are functions of position. The strain $e_{i j}$ and the increment $\xi$ can be expressed as

$$
e_{i j}=\frac{1}{2}\left(u_{i, j}+u_{j, i}\right), \xi=-w_{i, i}
$$

where $w_{i}=\phi\left(U_{i}-u_{i}\right)$ and $\phi$ is the porosity of the medium (a number between 0 and 1 ). The notation $u_{i, j}=\frac{\partial u_{i}}{\partial x_{j}}$ is used for brevity where applicable. The material coefficients $B_{1}$ to $B_{4}$ are spatially periodic functions determined by the material properties of the solid skeleton and fluid $^{41}$ :

$$
\begin{array}{r}
B_{1}=C_{44}, B_{2}=C_{12}+B_{3}^{2} / B_{4}, \\
B_{3}=-\left(1-\frac{1}{3 K_{s}}\left(C_{11}+2 C_{12}\right)\right) B_{4}, \\
B_{4}=\left(\frac{1-\phi}{K_{s}}+\frac{\phi}{K_{f}}-\frac{1}{3 K_{s}^{2}}\left(C_{11}+2 C_{12}\right)\right)^{-1},
\end{array}
$$

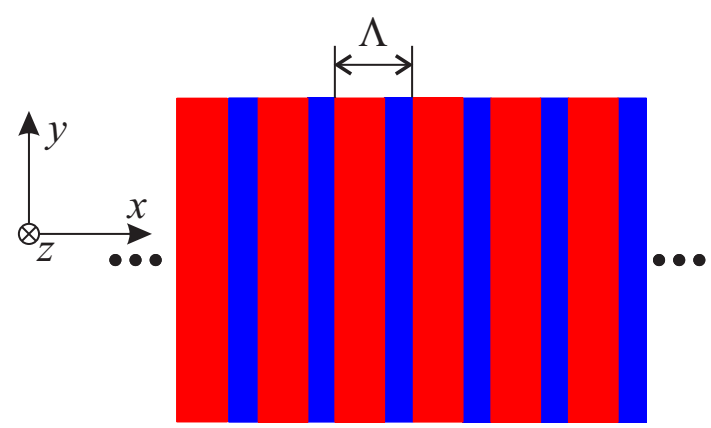

Figure 1: Sketch of the 1D fluid-saturated porous metamaterial with a periodic alternation of two layers. The lattice constant is $\Lambda$. Periodicity is along the $x$-direction; the other two directions are infinite.

where the $C_{i j}$ are the elastic constants of the solid skeleton, $K_{s}$ and $K_{f}$ are the bulk modulus of the solid skeleton and the pore fluid respectively.

The equations of motion can be written in Cartesian coordinates as

$$
\begin{array}{r}
\tau_{i j, j}=\rho \ddot{u}_{i}+\rho_{f} \ddot{w}_{i}, \\
-p_{, i}=\rho_{f} \ddot{u}_{i}+m_{i i} \ddot{w}_{i}+r_{i i} \dot{w}_{i},
\end{array}
$$

where $\rho=(1-\phi) \rho_{s}+\phi \rho_{f}, \rho_{f}$, and $\rho_{s}$ are the mass densities of the FSP media, the solid skeleton, and the pore fluid, respectively. $m_{i i}$ and $r_{i i}$ are coefficients introduced by Biot. For isotropic FSP materials, we have $m_{11}=m_{22}=m_{33}=m$ and $r_{11}=r_{22}=r_{33}=r$.

Without loss of generality, we limit our discussion to longitudinal motion restricted to the $x$-direction. For illustration, we consider an inhomogeneous FSPM with a periodic alternation of layers, as shown in Fig. 1. Combining the above equations, we have

$$
\begin{gathered}
\frac{\partial}{\partial x}\left[\left(2 B_{1}+B_{2}\right) \frac{\partial u_{x}}{\partial x}\right]-\frac{\partial}{\partial x}\left(B_{3} \frac{\partial w_{x}}{\partial x}\right)=\rho \ddot{u}_{x}+\rho_{f} \ddot{w}_{x}, \\
-\frac{\partial}{\partial x}\left(B_{3} \frac{\partial u_{x}}{\partial x}\right)+\frac{\partial}{\partial x}\left(B_{4} \frac{\partial w_{x}}{\partial x}\right)=\rho_{f} \ddot{u}_{x}+m \ddot{w}_{x}+r \dot{w}_{x}
\end{gathered}
$$

for longitudinal wave motion. Thus, the two independent variables are chosen as $\left(u_{x}, w_{x}\right)$. If at the interface between two FSP media longitudinal displacements are considered continuous, the open pore condition, then the natural boundary condition is the continuity of both normal stresses $\left(2 B_{1}+B_{2}\right) u_{x, x}-B_{3} w_{x, x}$ and $-B_{3} u_{x, x}+B_{4} w_{x, x}$.

\section{HARMONIC WAVE PROPAGATION}

\section{A. Harmonic waves}

For harmonic waves at angular frequency $\omega$, longitudinal displacements can be written

$$
\left[u_{x}, w_{x}\right]=\left[\bar{u}_{x}, \bar{w}_{x}\right] e^{-\imath \omega t},
$$


where $t$ is the time variable, and where $\bar{u}_{x}$ and $\bar{w}_{x}$ are functions of position $x$ otherwise independent of time. Here, we note $\mathbf{U}=\left(\bar{u}_{x}, \bar{w}_{x}\right)$. Substituting Eq. (5) into Eq. (4), we get

$$
\frac{\partial}{\partial x}\left(A_{0} \frac{\partial}{\partial x} \mathbf{U}\right)=-\rho \omega^{2} M_{0} \mathbf{U}
$$

with

$$
A_{0}=\left[\begin{array}{cc}
\left(2 B_{1}+B_{2}\right) & -B_{3} \\
-B_{3} & B_{4}
\end{array}\right], M_{0}=\left[\begin{array}{cc}
\rho & \rho_{f} \\
\rho_{f} & m_{1}
\end{array}\right]
$$

and $m_{1}=m+\imath r / \omega$.

The Biot coefficients $m$ and $r$ can be written ${ }^{28,36}$

$$
m=\operatorname{Re}[\alpha(\omega)] \rho_{f} / \phi, r=\operatorname{Re}[\eta / K(\omega)],
$$

where $\eta$ is the viscosity of the fluid, and $\alpha$ and $K$ are the dynamic tortuosity and permeability, respectively, with the relation:

$$
\alpha(\omega)=\imath \eta \phi /\left[K(\omega) \omega \rho_{f}\right] .
$$

For porous media with pores of simple form, the dynamic permeability can be expressed approximately as ${ }^{42}$

$$
\begin{aligned}
K(\omega)=K(0) & \left(\left[1-\frac{4 \imath \alpha^{2}(\infty) K^{2}(0) \omega \rho_{f}}{\eta d^{2} \phi^{2}}\right]^{1 / 2}\right. \\
& \left.-\frac{\imath \alpha(\infty) K(0) \omega \rho_{f}}{\eta \phi}\right)^{-1}
\end{aligned}
$$

where $d$ is the characteristic length of the pores. When the pores are a set of non-intersecting tubes, we further have

$$
8 \alpha(\infty) K(0) /\left(\phi d^{2}\right)=1
$$

\section{B. Bloch waves}

According to Bloch's theorem, the displacement field for propagation eigenmodes of a periodic medium is the product of a periodic function times a plane wave term. It thus can be written

$$
\mathbf{U}(x)=\mathbf{u}(x) e^{\imath k x},
$$

where $\mathbf{u}=\left(a_{x}, b_{x}\right)$, with $a_{x}$ and $b_{x}$ periodic amplitudes as a function of position, and $k$ is the Bloch wavenumber whose real part can be restricted to the first Brillouin zone of the reciprocal lattice. Substituting Eq. (11) into Eq. (6), we get

$$
\frac{\partial}{\partial x}\left(A_{0} \frac{\partial}{\partial x} \mathbf{u}\right)+2 \imath k A_{0} \frac{\partial}{\partial x} \mathbf{u}+\left(-k^{2} A_{0}+\omega^{2} M_{0}\right) \mathbf{u}=0
$$

for longitudinal wave motion.
In the present paper, numerical calculations are conducted with the finite element method. The layered system is treated as a periodic and inhomogeneous medium, whose material parameters are spatially periodic functions. The pores are chosen to be open at the interfaces, since the appearance of the P2 (slow) wave is restricted by the sealing of the pores at the interfaces ${ }^{43}$. The field variables $\left(a_{x}, b_{x}\right)$ between different layers are set as continuous ${ }^{44,45}$. We write the coefficient form of the resulting equation, suitable for use with the partial differential equation (PDE) module of Comsol Multiphysics for instance ${ }^{46}$, as

$$
\lambda^{2} A_{1} \mathbf{u}-\nabla \cdot\left(A_{0}: \nabla \mathbf{u}+A_{2} \mathbf{u}\right)+A_{3} \nabla \mathbf{u}+A_{4} \mathbf{u}=0
$$

where $\lambda=\imath k$ and the coefficient matrices are obtained from Eq. (12) as

$$
A_{1}=-A_{0}, A_{2}=-A_{3}=\lambda A_{0}, A_{4}=-\omega^{2} M_{0} .
$$

Since the dynamic material parameters of FSPMs are frequency-dependent, complex band structures ${ }^{46,47}$ are the first choice for the analysis. For this purpose, we set the eigenvalue as $\lambda=\imath k$. Complex band structures are then obtained by sweeping $\omega$ in the frequency range of interest.

In particular, if the pore fluid is lossless, i.e., $\eta=0$, we have $m=\alpha(\infty) \rho_{f} / \phi$ non dispersive and $r=0$ identically. Then $m_{1}=m$ and Eqs. (6) and (12) do not involve frequency-dependent coefficients. In this case, we may solve Eq. (12) for the real band structure by choosing the eigenvalue as $\lambda=\imath \omega$. The coefficient matrices of Eq. (14) are in this case

$$
A_{1}=M_{0}, A_{2}=-A_{3}=\imath k A_{0}, A_{4}=k^{2} A_{0} .
$$

The real band structure is then obtained by sweeping $k$ inside the first Brillouin zone of the 1D periodic metamaterial.

\section{Homogeneous FSP medium}

For homogeneous FSP media, the amplitudes $a_{x}$ and $b_{x}$ are constant functions, so the partial terms in Eq. (12) vanish. Then Eq. (12) reduces to

$$
\left(-k^{2} A_{0}+\omega^{2} M_{0}\right) \mathbf{u}=0 .
$$

Note that strickly speaking $k$ here denotes the wavenumber of a harmonic plane wave rather than a Bloch wavenumber and $\mathbf{u}$ is a constant vector representing the polarization of the plane wave. As a result, the dispersion relation for longitudinal harmonic plane waves can be obtained as

$$
k=\omega \sqrt{\frac{-b \pm \sqrt{b^{2}-4 a c}}{2 a}},
$$


Table I: Material parameters used in this paper. Units are given inside brackets.

\begin{tabular}{llllllllllll}
\hline Material & $\begin{array}{l}\rho_{s} \\
\left(\mathrm{~kg} / \mathrm{m}^{3}\right)\end{array}$ & $\begin{array}{l}\rho_{f} \\
\left(\mathrm{~kg} / \mathrm{m}^{3}\right)\end{array}$ & $\begin{array}{l}C_{11} \\
(\mathrm{GPa})\end{array}$ & $\begin{array}{l}C_{12} \\
(\mathrm{GPa})\end{array}$ & $\begin{array}{l}C_{44} \\
(\mathrm{GPa})\end{array}$ & $\begin{array}{l}K_{s} \\
(\mathrm{GPa})\end{array}$ & $\begin{array}{l}K_{f} \\
(\mathrm{GPa})\end{array}$ & $\begin{array}{l}\eta \\
(\mathrm{Pa} \cdot \mathrm{s})\end{array}$ & $\begin{array}{l}d \\
(\mu \mathrm{m})\end{array}$ & $\alpha(\infty)$ & $\phi$ \\
\hline Lossy FSP medium 1 & 3000 & 1000 & 10 & 2 & 4 & 30 & 2 & 0.001 & 6.32 & 1 & 0.2 \\
\hline Lossless FSP medium 1 & 3000 & 1000 & 10 & 2 & 4 & 30 & 2 & 0 & 6.32 & 1 & 0.2 \\
\hline Lossless FSP medium 2 & 2500 & 0.1 & 33.3 & 8.3 & 12.5 & 49.9 & 2 & 0 & 6.32 & 1 & 0.01 \\
\hline
\end{tabular}

where $a=B_{3}^{2}-\left(2 B_{1}+B_{2}\right) B_{4}, b=2 \rho_{f} B_{3}+\rho B_{4}+\left(2 B_{1}+\right.$ $\left.B_{2}\right) m_{1}$, and $c=\rho_{f}^{2}-\rho m_{1}$. Eq. (17) also suggests that there are two longitudinal waves in the porous medium, the sign + corresponding to the P1 (fast) wave and the sign - to the P2 (slow) wave. As a note, Eq. (4) could also be written with the fluid pressure $p$ replacing displacement $w_{x}$ as an independent variable ${ }^{41}$. In this case, exactly the same dispersion relation would be obtained, as we have checked. In this case, the eigenvectors defining the polarization would be different though the following results would still hold.

\section{Frequency response function}

The frequency response function $(\mathrm{FRF})^{48}$ of a finite system can also be calculated by solving Eq. (6), in order to compare with phononic band structures. The only non-zero coefficient matrices are in this case $A_{0}$ and $A_{4}=-\omega^{2} M_{0}$. Harmonic excitation with unit amplitude is applied to the left side of the finite system. Polarizations $\left(u_{x}, w_{x}\right)=(1,0)$ or $(0,1)$ are considered as excitations. Harmonic responses are measured at the right side of the system, and the frequency response function is defined as

$$
\mathrm{FRF}=-\log \frac{\sqrt{\bar{u}_{x}^{2}+\bar{w}_{x}^{2}}}{\sqrt{\bar{u}_{x 0}^{2}+\bar{w}_{x 0}^{2}}} .
$$

The imaginary part of the wavenumber characterizes the spatial decay of Bloch waves inside a $\mathrm{PC}^{47,49}$. As a result, the FRF can be approximated by using only the least evanescent Bloch wave ${ }^{50}$

$$
\mathrm{FRF} \approx-\log \left(T_{0} e^{-n \mid \operatorname{Im}(k(\omega) \mid \Lambda}\right)
$$

where $n$ is the number of layers, and $T_{0}$ is the conversion efficiency from the incident wave to the least evanescent Bloch wave.

\section{RESULTS AND DISCUSSION}

In this section, Bloch wave propagation in the 1D FSPMs is investigated, including complex band structures and harmonic responses. To get a convergent result, the size of the mesh is 100 times smaller than the lattice constant in the calculation of complex band structures, and 50 times smaller in the calculation of attenuation properties. Results for the homogeneous FSP media are first presented for reference and comparison.

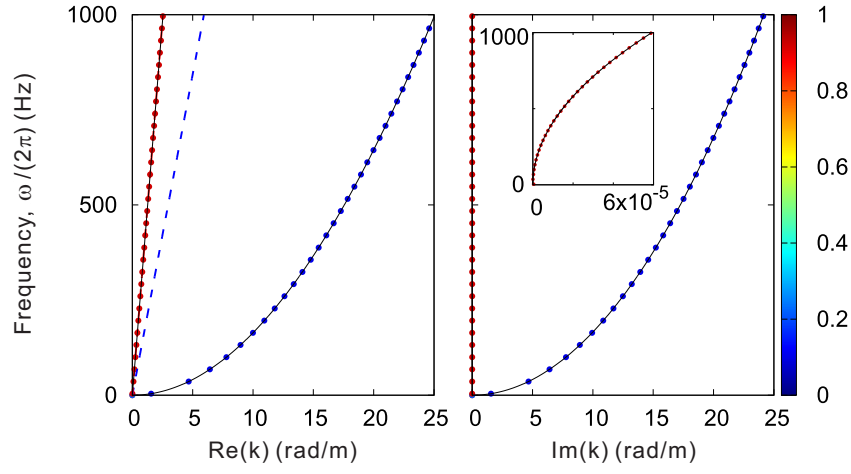

Figure 2: Complex band structures for homogeneous FSP media. The left and right panels illustrate the variation of the frequency with the real and imaginary part of the wave vector, respectively. The solid lines represent for the analytical results obtained by Eq. (17). The colorscale indicates the relative energy ratio between the solid skeleton (1) and the pore fluid (0). The blue and red dashed lines represent the analytical dispersion curves for lossless homogeneous media. The inset shows a closer view to the imaginary part at the origin.

\section{A. Homogeneous FSP media}

We first consider the case of a homogeneous FSP medium. The material parameters are listed in the second row of Table. I. It should be pointed out that the tortuosity is rigorously related to the electrical conductivity of the pore space ${ }^{51}$. We choose the ideal case $\alpha(\infty)=1$ for qualitative analysis ${ }^{42,45}$. The complex band structure $k(\omega)$ is shown in Fig. 2. It consists of two parts, showing the variations of frequency, $\omega /(2 \pi)$, as a function of the real $(\operatorname{Re}(k))$ and of the imaginary $(\operatorname{Im}(k))$ parts of the wavenumber in the direction of propagation. The colorscale amounts for the relative energy ratio between the kinetic energy $E_{k}^{f}$ for the pore fluid and the kinetic energy $E_{k}^{s}$ for the solid skeleton, defined as

$$
\frac{\int e_{k}^{s} d L}{\int\left(e_{k}^{s}+e_{k}^{f}\right) d L},
$$

where $L$ is the integration line along the unit cell, $e_{k}^{s}=$ $(1-\phi) \rho_{s} \omega^{2} u_{x}^{2} / 2$ and $e_{k}^{f}=\phi \rho_{f} \omega^{2} U_{x}^{2} / 2$. Both longitudinal waves are dispersive and lossy, as a result of the viscosity of the pore fluid. The polarization of the $\mathrm{P} 1$ wave is mainly $u_{x}$ while the polarization of the P2 wave is mostly $U_{x}$. The imaginary part of the wavenumber generally increases with frequency, but its value for the $\mathrm{P} 2$ wave is 

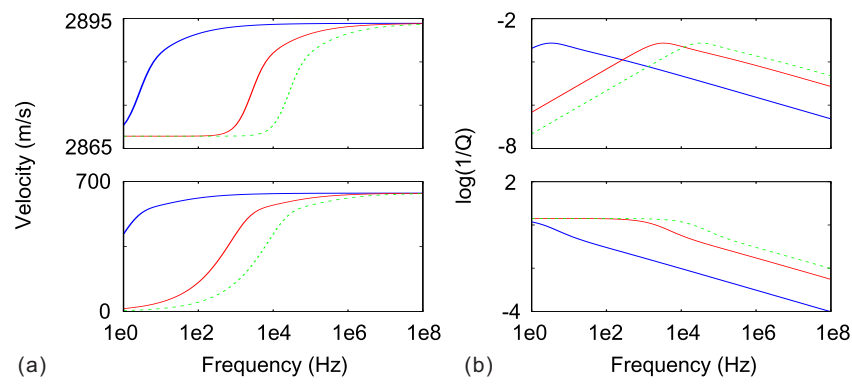

Figure 3: Variation of the phase velocity (a) and of the attenuation (b) with frequency for different viscosities of the pore fluid. The horizontal axis is shown with a logarithmic scale. The thick-solid blue, thin-solid red, and thin-dashed green lines represent the results for $\eta=10^{-7}, 10^{-4}$ and $10^{-3}$ $\mathrm{Pa} \cdot \mathrm{s}$, respectively. The upper and lower panels present the results for $\mathrm{P} 1$ and $\mathrm{P} 2$ waves, respectively.

much larger than that for the P1 wave, implying a much larger attenuation. The analytical result of Eq. (17) is in excellent agreement with the finite element result.

For comparison, the band structure for the lossless homogeneous FSP medium (third row of Table. I) is also plotted in Fig. 2. $\operatorname{Im}(k)$ is zero in this case for both waves, but $\operatorname{Re}(k)$ is almost unchanged for the $\mathrm{P} 1$ wave while it changes drastically for the $\mathrm{P} 2$ wave. In the low frequency range, the imaginary and the real parts of the wavenumber are nearly equal in the lossy case, in agreement with the results in Ref. ${ }^{45}$. We correspondingly evaluate in Fig. 3 the variation of phase velocity, $\omega / \operatorname{Re}(k)$, and of the attenuation, $\log (1 / Q)$, as a function of frequency. The quality factor is here defined as

$$
Q=\frac{\operatorname{Re}(k)}{2 \operatorname{Im}(k)} .
$$

As a note, this definition is slightly different from that used in Ref. ${ }^{28}$. Three different values of viscosity are considered in Fig. 3, $\eta=10^{-7}, 10^{-4}$ and $10^{-3} \mathrm{~Pa}$.s. The phase velocity of the $\mathrm{P} 1$ wave is not strongly affected by the value of viscosity and remains only slightly dispersive. The attenuation has larger variations with frequency: it first increases, reaches a maximum, and then decreases. The frequency of the maximum is termed the critical frequency and can be expressed as ${ }^{52}$

$$
f_{c}=3 \eta \phi /\left(8 \pi K(0) \alpha(\infty) \rho_{f}\right) .
$$

It is thus linearly proportional to viscosity. For the chosen values of viscosity, the critical frequency is $2.4 \mathrm{~Hz}$, $2.4 \mathrm{kHz}$, and $24 \mathrm{kHz}$, respectively. The phase velocity of the $\mathrm{P} 2$ wave, in sharp contrast, is strongly dependent on viscosity and strongly dispersive, with a sharp variation around the critical frequency. The attenuation remains almost constant until $f_{c}$ and then decreases with increasing frequency. For the same value of viscosity, the attenuation of the P2 wave is generally larger than that of the P1 wave.
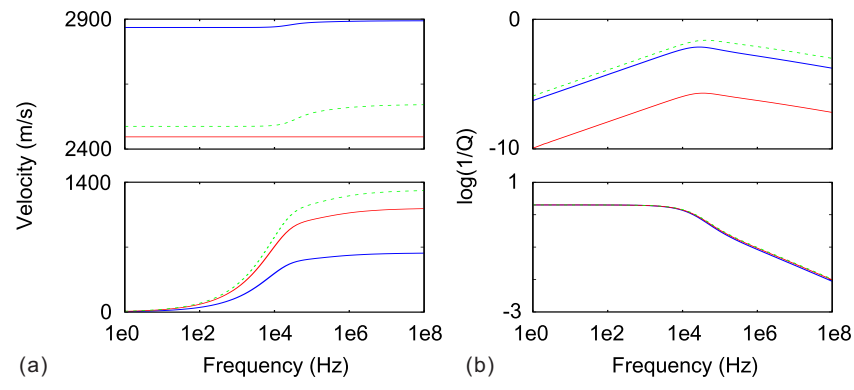

Figure 4: Variation of the phase velocity (a) and of the attenuation (b) with frequency for different values of porosity. The horizontal axis is shown with a logarithmic scale. The thick-solid blue, thin-solid red, and thin-dashed green lines present the results for $\phi=0.05,0.24$ and 0.45 , respectively. The upper and lower panels present the results for P1 and P2 waves, respectively.

It is furthermore instructive to consider the dependence of the complex dispersion with porosity in Fig. 4. In order to enable a fair comparison, the pore size $d$ is considered fixed. The change in porosity $\phi$ can then be understood as a change in the number of pores. According to Eq. (10), the critical frequency in Eq. (22) can be rewritten

$$
f_{c}=3 \eta /\left(\pi d^{2} \rho_{f}\right)
$$

This expression implies that $f_{c}$ is independent of porosity for a fixed pore size. It is found that porosity has a strong influence on both the phase velocity and the attenuation of the P1 wave. For the particular value $\phi=0.24$, the phase velocity is observed to be non dispersive. This effect is contained in the FSP model summarized in Section II, that is based on the literature, but has not yet been observed experimentally to the best of our knowledge. At a given frequency, the phase velocity and the attenuation first decrease and then increase with increasing porosity. The attenuation of the P2 wave is almost independent of porosity, but the asymptotic value of the phase velocity at large frequencies generally increases with porosity.

\section{B. 1D lossless FSP metamaterial}

We now turn our attention to wave propagation in $1 \mathrm{D}$ FSPM composed of periodic layers of FSP media. We first consider a lossless FSPM (zero viscosity). The filling ratio for FSP medium 2 is chosen to be 0.35 and the lattice constant is $\Lambda=2 \mathrm{~m}$. Material parameters are listed in the fourth row of Table I.

The complex band structure in Fig. 5(a-b) shows reduced frequency, $\Omega=\omega \Lambda /(2 \pi)$, as a function of reduced wavenumber, $k \Lambda /(2 \pi)$, as usual with PCs. We consider the reduced frequency range $0<\Omega<2000 \mathrm{~m} / \mathrm{s}$ and the corresponding frequency range is $0-1000 \mathrm{~Hz}$ for the chosen lattice constant. Given the color scale representing the energy ratio between the $\mathrm{P} 1$ and the $\mathrm{P} 2$ wave, it is 


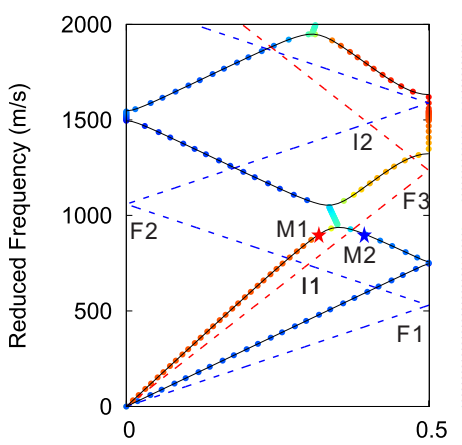

(a) $\operatorname{Re}(k \Lambda /(2 \pi))$

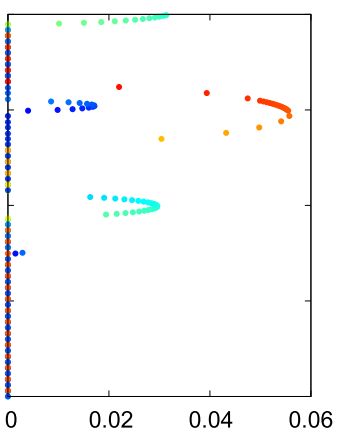

(b) $\quad \operatorname{Im}(\mathrm{k} \Lambda /(2 \pi))$

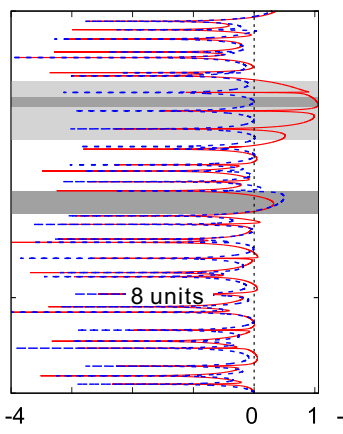

(c)

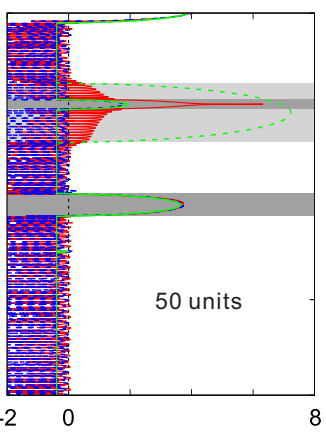

$\operatorname{FRF}(\mathrm{dB})$

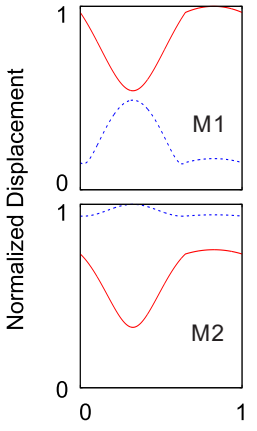

(d) $x / \Lambda$

Figure 5: Complex band structures for 1D lossless FSPM with periodic layers of FSP medium. Panels (a) and (b) illustrate the variation of the reduced frequency with the real and imaginary part of the wave vector, respectively. The solid lines represent the real band structures obtained by using Eqs. (13) and (15). The blue and red dashed lines are the dispersion curves of P1 and P2 waves for lossless FSP media, as obtained by Eq. (17). The colorscale is the same as that in Fig. 2. FRF curves of a finite metamaterial with 8 or 50 unit cells are shown in panel (c). The solid and dashed lines represent the results for the excitation of P1 and P2 waves, respectively. The light gray region indicates the Bragg bandgap for P1 wave in panel (a), while the gray regions show the complete bandgaps for both $\mathrm{P} 1$ and $\mathrm{P} 2$ waves. The green lines represent the results predicted by using Eq. (19) through the imaginary part of the wave vector for P1 wave (dashed lines for P2 wave). Normalized displacement distributions at the marked points in panel (a) are illustrated in panel (d). The relative energy ratio is 0.74 at point M1 and 0.27 at point M2.

inferred that both longitudinal waves exist in the composite metamaterial and can form Bragg band gaps opening at the edges of the first Brillouin zone. The Bragg band gap for the P1 wave is the widest and contains the second Bragg band gap for the P2 wave. The first Bragg band gap for the P2 wave is too narrow to be observed from the real part of the wavenumber alone but still can be clearly identified from the imaginary part of the wavenumber. Overall, the complete Bragg bandgap is small and covers the reduced frequency range $1495<\Omega<1547 \mathrm{~m} / \mathrm{s}$.

Since the polarizations of P1 and P2 waves share coupled longitudinal displacements, they can interfere whenever their dispersion curves cross. As a result of band anti-crossing, two complete bandgaps appear around $\Omega \sim$ 1000 and $2000 \mathrm{~m} / \mathrm{s}$ in Fig. 5(a-b). Vibration distributions at the marked points M1 and M2 near the first anti-crossing region are presented in Fig. 5(d). It is noted that the polarizations of these Bloch waves change abruptly at the anti-crossing, as can be attributed to level repulsion or avoided-crossing of the bands ${ }^{53,54}$. A pair of evanescent Bloch waves appear inside the anticrossing bandgaps, connecting the extremal points of the two dispersion bands. The attenuation of the P1 and P2 waves are identical inside these bandgaps, implying the bandgaps are complete.

In addition, the FRF curves of a finite metamaterial are calculated and presented in Fig. 5(c) for either 8 or 50 periods. Many oscillations come up in the FRF curves, even inside bandgaps. These oscillations are caused by the excitation of Fabry-Perot resonances of the finite structure. Since the polarization of the P1 and P2 waves share coupled longitudinal displacements, the resonances affect both waves simultaneously. Bragg bandgaps can not be clearly observed when only 8 periods are consid- ered. For 50 periods, the first P2 Bragg bandgap is still missing in the FRF curves, because the imaginary part of the wavenumber remains very small. The Bragg bandgap for the P1 wave is corrupted by Fabry-Perot resonances of the P2 wave. Interestingly, the anti-crossing bandgaps show the same attenuation for P1 and P2 waves, in agreement with the complex band structure. The approximation of the FRF curves inside bandgaps as obtained with Eq. (19) are added with a green line to Fig. 5(c). They are in excellent agreement with FEM results in all complete bandgaps. Inside the Bragg bandgap for the P1 wave, the approximation breaks when wave P2 is not itself inside a bandgap. Again, this is attributed to the coupling of longitudinal waves in the solid skeleton and in fluid saturated pores.

\section{Effects of fluid viscosity}

Next, we consider FSP metamaterials with varying viscosity, as shown in Fig. 6. Even when only a slight viscosity is added $\left(\eta=10^{-7} \mathrm{~Pa} \cdot \mathrm{s}\right)$, the dispersion curves are affected compared to the lossless case of the previous section. Regarding the real part of the wavenumber, the sharp corners at the edge of the Brillouin zone for the $\mathrm{P} 1$ wave become rounded. The degeneration of the evanescent modes at the anti-crossings is now lifted for the imaginary part of the wavenumber. The P2 wave presents a larger attenuation than the P1 wave, in correspondance to the larger attenuation of the P2 wave in a homogeneous FSP medium shown in Fig. 3. FRF curves are also affected by viscosity. The Fabry-Perot oscillations of the lossless system are washed out, especially inside the Bragg bandgap for the P1 wave. The atten- 

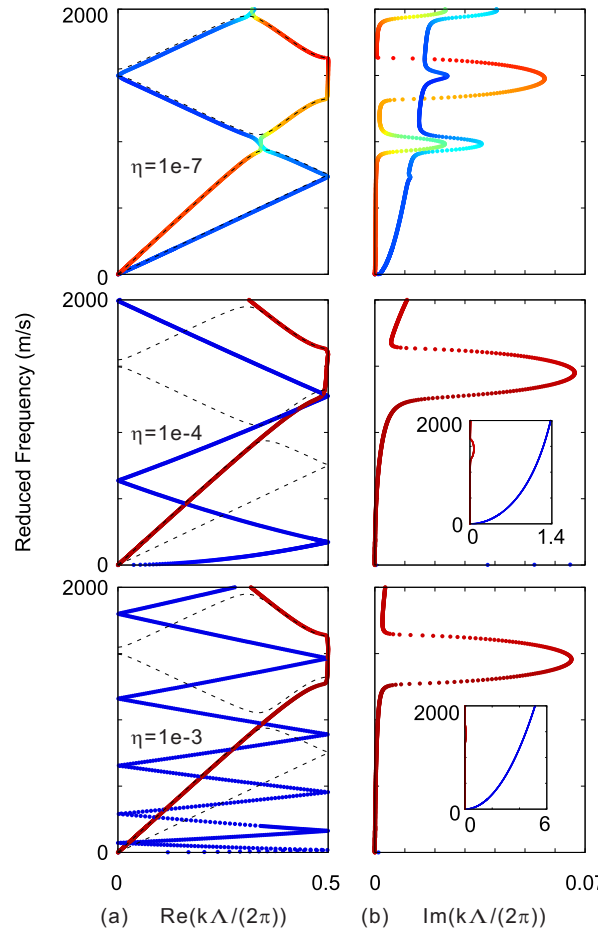

(b) $\operatorname{Im}(k \Lambda /(2 \pi))$
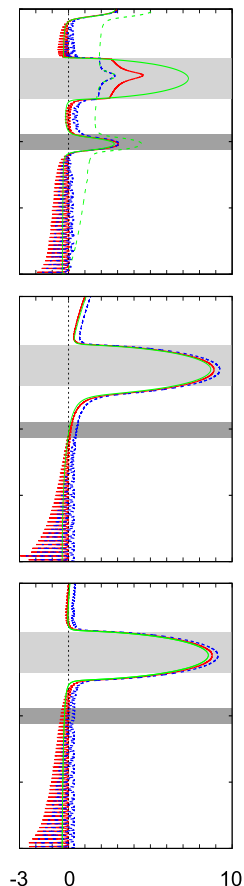

(c) $\quad \mathrm{FRF}(\mathrm{dB})$

Figure 6: Influence of fluid viscosity on complex band structures for 1D FSP metamaterials. Panels (a) and (b) show the variation of the reduced frequency with the real and imaginary part of the reduced wavenumber. The colorscale is the same as that in Fig. 2. The insets in (b) show a larger view of the imaginary part of the wavenumber. The attenuation properties of a finite metamaterial with 50 periods are shown in panel (c). The red solid and blue dashed lines represent the results for the excitation of $u_{x}$ and $w_{x}$, respectively. The green lines represent the results predicted by Eq. (19) for the $\mathrm{P} 1$ wave (green dashed lines for the P2 wave). The gray areas indicate the bandgaps for the lossless FSPM in Fig. 5.

uation is generally larger for all frequencies and adds to the attenuation already present inside the bandgaps.

When $\eta=10^{-4} \mathrm{~Pa} \cdot \mathrm{s}, f_{c}$ is beyond the frequency range considered and the attenuation of the P2 wave is expected to be quite large following Fig. 3. Thus, the dispersion relation of the P2 wave resembles that of the homogeneous FSP medium in Fig. 2. The anti-crossings disappear from the FRF, as the P2 wave is too much attenuated to interfere with the $\mathrm{P} 1$ wave. The Bragg bandgaps for the P2 wave also disappear. When the viscosity is further increased to $\eta=10^{-3} \mathrm{~Pa} \cdot \mathrm{s}$, the same observations amplify. Interestingly, the base attenuation for the P1 wave (outside the Bragg bandgap) is smaller than for $\eta=10^{-4} \mathrm{~Pa} \cdot \mathrm{s}$. This observation is in correspondence with the decrease of the attenuation of the P1 wave in Fig. 3(b) for larger viscosities. With an increase in viscosity, the number of foldings for the P2 wave generally increases, but the same number for the P2 wave is almost unchanged. This could also be explained by the distinct variation of phase velocity of the P2 wave with a change in viscosity indicated by Fig. 3(a). For both
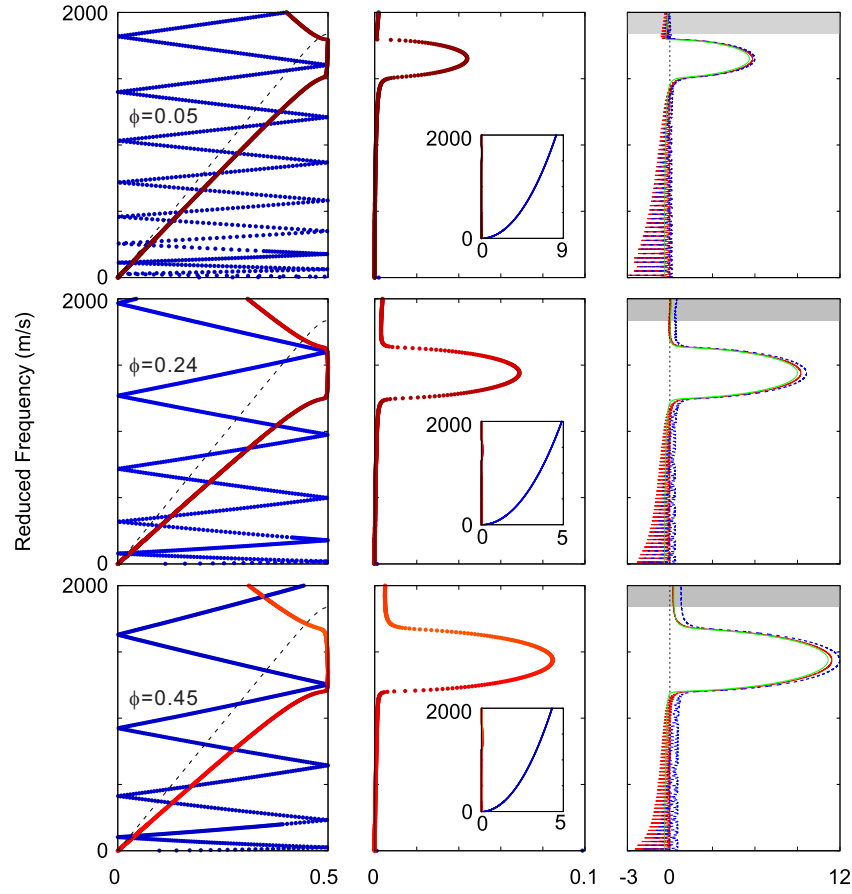

(a) $\operatorname{Re}(\mathrm{k} \Lambda /(2 \pi))$

(b) $\operatorname{Im}(\mathrm{k} \Lambda /(2 \pi))$

(c) $\quad F R F(d B)$

Figure 7: Influence of porosity on the complex band structures for 1D FSPMs. Panels (a) and (b) show the variation of the reduced frequency with the real and imaginary parts of the reduced wavenumber. The dashed lines are the dispersion curves for $\phi=0$. The colorscale is the same as that in Fig. 2. FRF curves for a finite metamaterial with 50 periods are shown in panel (c). The red solid and blue dashed lines represent the results for the excitation of $u_{x}$ and $w_{x}$, respectively. The green lines present the results of Eq. (19) for the $\mathrm{P} 1$ wave.

excitations, the FRF curves have only small differences at large viscosity. Generally, the transmission of the P1 wave dominates the frequency response. As a result, the FRF curves can be closely approximated by considering only the least evanescent P1 wave.

It is also noted that the curvature of the lower round corner of the Bragg bandgap for P1 wave first increases and then decreases. This can be simply explained by expanding $\omega(k)$ in powers of complex $k$ about the zone edge $k_{0}=\pi / d$ via a Taylor expansion ${ }^{55}$, i.e.

$$
\Delta \omega=\omega(k)-\omega\left(k_{0}\right) \approx \zeta \operatorname{Re}\left((\Delta k)^{2}\right)=\zeta\left(g^{2}-h^{2}\right),
$$

where $\zeta$ is a constant related to the second derivative of the band, and $\Delta k=g+\imath h$. For the same $\Delta \omega>0$ and a different viscosity, since the attenuation in Fig. 3(b) first increases and then decreases, the corresponding imaginary part first increases then decreases. Then $h$ follows a similar variation, and so does $g$ according to Eq. (24). Hence, the corners first get rounded and then become sharper. 


\section{Effects of fluid porosity}

Porosity is also an important factor for FSP media. In this section, we consider the influence of fluid porosity on the complex band structures and the attenuation spectra. For comparison, we also consider the system with zero porosity. In this case, the system reduces to a $1 \mathrm{D}$ elastic layered metamaterial, the band structure of which is plotted with a dashed line in Fig. 7; no P2 wave exists in this case. Even when a small porosity $(\phi=0.05)$ is considered, the P2 wave comes up and the phase velocity of the P1 wave decreases. Correspondingly, the Bragg bandgap for the P1 wave shifts downward. Comparing with Fig. 4, with an increase in porosity, the phase velocity of the $\mathrm{P} 2$ wave increases monotonously and there are less foldings in the real part of the P2 wave. In contrast, the phase velocity of the $\mathrm{P} 1$ wave first decreases and then increases, but the relative changes remain small. The real parts for $\phi=0.24$ and 0.45 are thus similar. Both outside and inside the Bragg bandgap, the imaginary wavenumber of the P1 wave increases monotonously with porosity, leading to an overall lower transmission. This observation is in contrast with the variations of attenuation for the $\mathrm{P} 1$ wave in a homogeneous FSP medium shown in Fig. 4(b). We attribute this difference to the periodicity of the FSPM. It is also noted that the upper (lower) corner of the bandgap generally becomes more rounded (sharper) with an increase in the fluid porosity. This variation is explained by Eq. (24) as in the case of viscosity.

\section{CONCLUSIONS}

This paper has presented a comprehensive analysis of wave propagation in 1D fluid-saturated porous metamaterials (FSPMs) described by Biot's model and supporting two longitudinal waves, P1 and P2. Complex band structures and harmonic responses of 1D FSPMs were calculated by a finite element method. A theoretical analysis of longitudinal wave motion in homogeneous
FSP media was conducted. Numerical results match perfectly analytical ones for homogeneous FSP media. It is found that the material parameters of the pore fluid have a strong influence on both complex band structures and FRF curves. With an increase (decrease) in viscosity (porosity), the attenuation in the passing bands is first enhanced and then reduced. Inside Bragg bandgaps, the attenuation varies in the opposite way. Anti-crossing bandgaps can be generated by level repulsion between the fast P1 wave and the slow P2 wave when viscosity is negligible. These anti-crossing bandgaps soon disappear, however, as viscosity increases. With an increase in fluid porosity, attenuation increases monotonously inside passing bands and Bragg bandgaps.

Generally speaking, the P2 wave is highly attenuated and influenced by material parameters. The P1 wave then plays the key role in the transmission though a finite periodic structure. In the limit that the influence of the P2 wave has been removed by viscosity, the frequency response is well predicted by considering only the least evanescent P1 wave.

The present work is limited to 1D periodic metamaterials and should be extended to the $2 \mathrm{D}$ and $3 \mathrm{D}$ cases. In this case, appropriate independent variables should be chosen, as there are 6 displacements but only 4 degrees of freedom ${ }^{41}$. The influence of other complex interface conditions on wave propagation in FSPMs should be discussed ${ }^{56}$. The case of surface waves $^{57,58}$ propagating over a fluid-saturated porous metamaterial is also of interest. These different problems are significant for the development of novel metamaterials applied to the mitigation of blast and/or seismic waves.

\section{ACKNOWLEDGMENTS}

Financial support by the National Natural Science Foundation of China (11702017 and 11532001) is gratefully acknowledged. V.L. acknowledges financial support by the EIPHI Graduate School (ANR-17-EURE-0002).
* Electronic address: yswang@tju.edu.cn

† Electronic address: vincent.laude@femto-st.fr

1 S. Prakash, Soil Dynamics (McGraw-Hill, New York, 1981).

2 D. K. S. Ashwanijain, in Proceedings of 3rd WSEAS International Conference on Applied and Theoretical Mechanics (2007).

3 K. A. Kuo and H. E. M. Hunt, Applied Mechanics Reviews 65, 031003 (2013).

4 A. Niousha and M. Motosaka, Advances in Earthquake Engineering 9, 191 (2001).

${ }^{5}$ M. S. Kushwaha, P. Halevi, L. Dobrzyński, and B. DjafariRouhani, Physical Review Letters 71, 2022 (1993).

${ }^{6}$ M. I. Hussein, M. J. Leamy, and M. Ruzzene, Applied Mechanics Reviews 66, 040802 (2014).
7 Z. Liu, X. Zhang, Y. Mao, Y. Y. Zhu, Z. Yang, C. T. Chan, and P. Sheng, Science 289, 1734 (2000).

${ }^{8}$ Z. Liu, C. T. Chan, and P. Sheng, Physical Review B 71, 014103 (2005).

${ }^{9}$ G. Ma and P. Sheng, Science Advances 2, e1501595 (2016).

10 M. S. Kushwaha and P. Halevi, Applied Physics Letters 64, 1085 (1994).

${ }^{11}$ G. F. Jia and Z. F. Shi, Earthquake Engineering and Engineering Vibration 9, 75 (2010).

12 A. Malcolm and D. P. Nicholls, Journal of the Acoustical Society of America 129, 1783 (2011).

13 J. Bao, Z. F. Shi, and H. J. Xiao, Journal of Engineering Mechanics 138, 761 (2012).

14 S. H. Kim and M. P. Das, Modern Physics Letters B 26, 1250105 (2012). 
15 Z. F. Shi and J. K. Huang, Soil Dynamics and Earthquake Engineering 50, 204 (2013).

16 S. J. Mitchell, A. Pandolfi, and M. Ortiz, Journal of the Mechanics and Physics of Solids 65, 69 (2014).

17 A. Colombi, P. Roux, S. Guenneau, P. Gueguen, and R. V. Craster, Scientific Reports 6, 19238 (2016).

18 D. J. Colquitt, A. Colombi, R. V. Craster, P. Roux, and S. R. L. Guenneau, Journal of the Mechanics and Physics of Solids 99, 379 (2017).

19 S. Brûlé, E. H. Javelaud, S. Enoch, and S. Guenneau, Physical Review Letters 112, 133901 (2014).

20 Y. Yan, A. Laskar, Z. Cheng, F. Meng, Y. Tang, Y. L. Mo, and Z. F. Shi, Journal of Applied Physics 116, 044908 (2014).

21 A. Colombi, D. Colquitt, P. Roux, S. Guenneau, and R. V. Craster, Scientific Reports 6, 27717 (2016).

${ }^{22}$ H. Franklin, F. Luppé, and J. M. Conoir, Journal of the Acoustical Society of America 135, 2513 (2014).

23 T. Weisser, J.-P. Groby, O. Dazel, F. Gaultier, E. Deckers, S. Futatsugi, and L. Monteiro, Journal of the Acoustical Society of America 139, 617 (2016).

24 A. Alevizaki, R. Sainidou, P. Rembert, B. Morvan, and N. Stefanou, Physical Review B 94, 174306 (2016).

25 A. Alevizaki, R. Sainidou, P. Rembert, B. Morvan, and N. Stefanou, Physical Review B 95, 214306 (2017).

${ }^{26}$ W. Trabelsi, H. Franklin, A. Tinel, and S. Derible, Ultrasonics 54, 1097 (2014).

${ }^{27}$ H. Deresiewicz, Bulletin of the Seismological Society of America 52, 627 (1962)

${ }^{28}$ R. D. Stoll and G. M. Bryan, Journal of the Acoustical Society of America 47, 1440 (1970).

29 B. R. Simon, O. C. Zienkiewicz, and D. K. Paul, International Journal for Numerical and Analytical Methods in Geomechanics 8, 381 (1984).

30 A. Nur, Wave propagation in the two-phase media (Petroleum Industry Press, Beijing, 1986).

31 M. A. Biot, Journal of the Acoustical Society of America 28, 168 (1956)

32 M. A. Biot, Journal of the Acoustical Society of America 28, 179 (1956).

33 T. J. Plona, Applied Physics Letters 36, 259 (1980).

34 N. C. Dutta, Applied Physics Letters 37, 898 (1980).

35 J. M. Carcione, Journal of the Acoustical Society of America 99, 2655 (1996).

${ }^{36}$ Y. S. Wang and Z. M. Zhang, Journal of the Acoustical Society of America 103, 695 (1998).

37 L. De Ryck, J. P. Groby, P. Leclaire, W. Lauriks, A. Wirgin, Z. E. A. Fellah, and C. Depollier, Applied Physics
Letters 90, 181901 (2007).

38 Y. Liu and L. T. Gao, International Journal of Solids and Structures 45, 4860 (2008).

39 A. Pooladi, M. Rahimian, and R. Y. S. Pak, Applied Mathematical Modelling 50, 177 (2017).

${ }^{40}$ Y. Liu, K. Liu, L. T. Gao, and T. X. Yu, Journal of Sound and Vibration 282, 863 (2005).

41 M. N. Kazi-Aoual, G. Bonnet, and P. Jouanna, Journal of the Acoustical Society of America 84, 1883 (1988).

42 D. L. Johnson, J. Koplik, and R. Dashen, Journal of Fluid Mechanics 176, 379 (1987).

43 P. N. J. Rasolofosaon, Applied Physics Letters 52, 780 (1988).

44 D. L. Johnson, T. J. Plona, and H. Kojima, Journal of Applied Physics 76, 115 (1994).

45 J. Allard and N. Atalla, Propagation of sound in porous media: modelling sound absorbing materials (second edition) (John Wiley \& Sons, United Kingdom, 2009).

46 Y.-F. Wang, Y.-S. Wang, and V. Laude, Physical Review B 92, 104110 (2015).

47 V. Laude, Y. Achaoui, S. Benchabane, and A. Khelif, Physical Review B 80, 092301 (2009).

48 J. S. Jensen, Journal of Sound and Vibration 266, 1053 (2003).

49 V. Romero-García, J. V. Sánchez-Pérez, S. CastiñeiraIbáñez, and L. M. Garcia-Raffi, Applied Physics Letters 96, 124102 (2010)

50 V. Laude, Phononic Crystals: Artificial Crystals for Sonic, Acoustic, and Elastic Waves (Walter de Gruyter GmbH, Berlin, 2015).

51 D. L. Johnson, T. J. Plona, C. Scala, F. Pasierb, and H. Kojima, Physical Review Letters 49, 1840 (1982).

52 D. P. Schmitt, Journal of the Acoustical Society of America 86, 2397 (1989).

53 D. L. Johnson and T. J. Plona, Journal of the Acoustical Society of America 72, 556 (1982).

54 T.-T. Wu and Z.-G. Huang, Physical Review B 70, 214304 (2004).

55 J. D. Joannopoulos, S. G. Johnson, J. N. Winn, and R. D. Meade, Photonic Crystals: Modeling the Flow of Light, 2nd ed. (Princeton University Press, New Jersey, 2008).

${ }^{56}$ H. Deresiewicz and R. Skalak, Bulletin of the Seismological Society of America 53, 783 (1963).

57 K. Ahn, Y. A. Kosevich, and M. W. Kim, Europhysics Letters 60, 241 (2002).

58 Y. A. Kosevich, K. Ahn, and M. W. Kim, Physical Review Letters 90, 059601 (2003). 